\title{
A SHORT HISTORICAL OVERVIEW OF MALARIA IN WORLDWIDE AND IN BULGARIA. THE FIGHT AGAINST MALARIA - THE MAIN TASK OF SANITARY EPIDEMIOLOGICAL STATION IN STARA ZAGORA IN THE FIRST YEAR OF ITS OPENING, 1950
}

\author{
B. Chakarova ${ }^{1^{*}}$, K. Kichukova ${ }^{2}$, G. Karaslavov ${ }^{3}$ \\ ${ }^{1}$ Department of Hygiene, Epidemiology Infectious and Parasitic Diseases, Faculty of Medicine, \\ Trakia University, Stara Zagora, Bulgaria \\ ${ }^{2}$ Medical College, Trakia University, Stara Zagora, Bulgaria \\ ${ }^{3}$ State Archive, Stara Zagora, Bulgaria
}

\begin{abstract}
Malaria is a mosquito-borne life-threatening infectious disease of humans, caused by parasites (genus: Plasmodium) that are transmitted to people through the bites of infected mosquitoes (genus: Anopheles). The risk of disease can be reduced by timely treatment of infected people (sources of disease), preventing mosquito bites by using mosquito nets and insect repellents, or with mosquitocontrol measures such as spraying insecticides and draining standing water. The Global Fund to Fight AIDS, Tuberculosis and Malaria has distributed 230 million insecticide-treated nets intended to stop mosquito-borne transmission of malaria. During the first half of the 20th century, malaria commanded the attention of a cohort of outstanding researchers and clinicians. The fight against malaria becomes a priority and one of the main tasks during the first years of the discovery (in 1950) the sanitary epidemiological stations in Bulgaria respectively in Stara Zagora. There have been systematic and planned activities: irrigation events, controlled irrigation, limiting the area of rice fields in order to limit the areas of mosquitoes of the genus Anopheles vectors of malaria, treatment with insecticides and DDT, perform active prevention of malaria by conducting research, treatment active surveillance of infected and recurrent cases. As a result of activities in 1965, WHO certified Bulgaria as eliminated autochthonous malaria.
\end{abstract}

Key words: autochthonous malaria, sanitary epidemiological stations, Bulgaria, Stara Zagora-region, 1950

Short historical data for the malaria

Malaria remains one of the major killers of humans worldwide, threatening the lives of more than $1 / 3$ of the world's population [1]. There is a suggestion that people have been suffering from malaria for the past 5,000 years. It is believed that its homeland is West Africa malaria ( $P$. falciparum) and Central Africa $(P$. vivax). The first chronicles evidencing fever caused by malaria, have been found in China (about 2700 BC the year, the time of the Xia Dynasty). The name is derived from the Italian, "mal aria," or bad air [2]. Four

\footnotetext{
*Correspondence to: Borislava Georgieva Chakarova, Department of Hygiene, Epidemiology, Infectious \& Parasitic Diseases, Faculty of Medicine, Trakia University, 6000 Stara Zagora, Bulgaria, Phone (++359 42) 664 233, Cell phone ++359888 871 601, e-mail: borislava_chakarova@abv.bg
}

species of malaria parasite infect humans: $P$. falciparum, $P$. vivax, $P$. malariae, $P$. ovale [3]. A widespread and potentially lethal human infectious disease, at its peak malaria infested every continent, except Antarctica $[4,5]$.

Malaria in the world - current situation
According to the World Health
Organization1 (WHO), approximately 660,000 people died from malaria in 2010 globally, and 584000 deaths - in 2013. There were an estimated 219 million cases of malaria infection in 2010 worldwide. Mortality rates have fallen by over $25 \%$ since 2000. The malaria burden in many parts of the world is being dramatically reduced thanks to increased malaria prevention and control measures. About $80 \%$ of all malaria cases occur in just 17 countries. About 1,500 people are diagnosed in the U.S. with malaria each year. The vast majority were infected abroad [6]. 


\section{Malaria in Europe}

A campaign launched at the end of the 1950s eradicated malaria in all countries in the WHO European Region. By the 1980s, malaria was an almost forgotten disease. In recent years it has dramatically re-emerged. There has been an eight-fold increase in imported cases since the 1970s: 1500 cases were reported in 1972, 13000 cases in 1999. The 51 countries in the WHO European Region are located in an area corresponding geographically to Europe, Anatolia, the Caucasus, Siberia, and central Asia [7,8].

\section{Autochthonous malaria in European Region}

Since the early 1990 s, the malaria situation has deteriorated considerably in some of the countries of the WHO European Region, owing to political and economic instability, massive population movements, and large scale hydro-agricultural projects. Experience shows that malaria can spread rapidly and is a continual threat. For instance, Greece had managed to remain malaria free between 1974 and 2009, but in 2010 three locally acquired malaria cases were reported, followed by 40 in 2011, 20 in 2012 and three in 2013. Following intensified control efforts, local malaria transmission in Greece was interrupted and zero locally acquired cases were reported in the country in 2014 [9]. In recent years, Azerbaijan, Tajikistan, and Turkey have had massive epidemics, whereas Armenia, Turkmenistan, and Georgia experienced small outbreaks. Sporadic cases of endemic malaria were also reported in Kazakhstan, Kyrgyzstan, the Russian Federation, Uzbekistan, Moldova, Bulgaria (1995 - 7 and 1996 - 11 cases), Greece, and Italy. In 1995, a total of 90712 cases of endemic malaria were reported in the region. In 1996-1999, the reported number of cases declined from about 90506 to 37170 , and it is expected that the malaria cases will slightly increase to about 42000 in 2000 [10].

The last report of ECDC (2014) [11]. presents the epidemiological situation for emerging and vector-borne diseases as of 2012. The confirmed case rate of malaria reported by $25 \mathrm{EU}$ and one EEA countries in continental Europe remained stable, fluctuating around one per $100 \quad 000$ population, with a slight reduction in 2012 compared with 2011 and 2010. Eighty-five per cent of the cases were reported by five countries: France, United Kingdom, Germany, Spain and Belgium. Highest rates of confirmed cases were reported by the United Kingdom, Belgium, Ireland and Luxembourg. The overall confirmed case rate was 0.88 per 100000 population in 2012. The individual country rates varied between 0.05 (Hungary and Poland) and 2.19 cases (United Kingdom) per 100000 population. Ninety nine $\%$ of cases (where origin is specified) were imported; these were reported by EU and EEA countries that have strong traditional ties with endemic areas. Twenty-six cases were reported as not-imported of which twenty-two were from Greece, three from Belgium and one from France. Eighteen of the twenty-two cases reported from Greece were attributed to the 2012 transmission season, whereas four cases were attributed to the previous transmission season. The one case reported from France was of Plasmodium falciparum malaria transmitted through red blood cell transfusion. Presence of Plasmodium falciparum in the donor could be evidenced only by a positive PCR test (France, personal communication). Autochthonous transmission of malaria has occasionally been reported over the last 10 years [12, 13, 14]. Greece reported for the fourth year autochthonous transmission of Plasmodium vivax malaria: local cases of malaria have occurred in Greece since 2009 with the highest number reported in 2011, $\mathrm{n}=20$. In 2012 local malaria transmission still took place but fewer cases were reported, with the municipality of Evrotas as the most affected locality. In addition to the data available in this report, Italy reported two cases of probable autochthonous introduced malaria in 2009-2011 [15].

\section{Malaria in Bulgaria}

In the past, malaria in Bulgaria was one of the most serious social diseases, known as „Thracian faver" [16]. Malaria had been endemic since ancient times. It was widespread in areas located in the valleys of major rivers, their tributaries and along the Black Sea coast. In 1919 in Bulgaria created a special law for fight against malaria. In 1928 in Burgas a special institute for control and conducting of remedial antimalarial measures was established, and in 1930 in Petrich, with the help of the Rockefeller Foundation an Experimental antimalarial station was built [17]. The highest incidence was recorded in 1946 - 144632 cases (2066\%) [18]. According to K. Markov et al., (1947) annually over 200,000 people get sick of malaria and between 200 and 300 people dieAfter 1944, the Ministry of Health established a department for fight against 
malaria. In 1948, the country started its malaria eradication campaign, in line with the WHO eradication programme [17, 19]. It included control of mosquitoes, detection and treatment of malaria cases and mass administration of medicines.. At this time there have been two antimalarial Institutes in the country - in Burgas and in Varna, and 37 antimalarial stations [17]. Ministry of Public Health develops methodical guidance concerning organization of systematic fight against malaria, especially: (1) in the conduct of treatment to limit recurrences in malaria; (2) treatment with DDT; (3) and implementation of remedial actions [20-24]. In 1950 began a planned fight with malaria aiming at its eradication. For almost 20 years a prophylactic network in Bulgaria and Stara Zagora region has been working on the implementation of Decree 2521 (September 22, 1950) for fight against malaria in Bulgaria. In the first year of its opening, the Station has been actively working on the implementation of the Instruction "Antimalarial requirements for the sowing of rice and organization of new rice fields".

As a result of complex anti-malarial activities and efforts of government, and public health organizations in 1964 eradication of malaria on the whole territory of country was achieved. In July 1965, Bulgaria was certified malaria free by WHO [19]. From 1966 to 2014, there were nearly 3000 cases of malaria of all types imported into Bulgaria, resulting in 16 deaths $[3,11$, $17,19,25,26]$. For the first time after the malaria eradication in 1995-1996, some cases have been diagnosed in southeast Bulgaria, near the border with Greece and Macedonia - 18 autochthonous cases of malaria tertiana $(P$. vivax) and its vector Anopheles macculipennis. Most probably the origin of the parasite carrier of $P$. vivax comes from African immigrants that has been in this area in June - July 1995 on his way to Greece [18].

On 29 May 2015, Bulgaria celebrated 50 years of being malaria-free. This is the result of the enormous efforts of several generations of health workers, who first combated malaria and then managed to keep the country free of the disease. The Ministry of Health of Bulgaria, the National Centre of Infectious and Parasitic Diseases and the Bulgarian Society for Parasitology are hosting the anniversary event [19].

The fight against malaria - the main task of Sanitary epidemiological station in
Stara Zagora in the first year of its opening, 1950

Development of sanitary network in Stara Zagora began in 1-th January, 1950. Then a Sanitary and Epidemiological Service was founded. After the release of the Decree of the Presidium of the National Assembly in 1951, the Service was transformed into sanitary-epidemiological station. There are detailed instructions on: (1) Choice of territory and conducting detailed studies; (2) Designing an irrigation system of rice fields and carrying out the construction work; (3) Conducting antilarval measures in the rice fields.

From January 1, 1951 in Stara Zagora region there were 8 counties, of which 4 - Nova Zagora, Sliven, Yambol and Topolovgrad with high endemicity of malaria. Malaria area of the county covered 101 villages declared malaria, with about 150000 inhabitants. Total suffered from malaria were 4099 persons and prophylactic treatment (pre-season) were conducted 2336 persons. In 1950 in Stara Zagora for malaria were tested a total of 193117 people from 103 settlements. Positive for malaria were $621(0.32 \%)$ persons. Of them infected with $P$. vivax were $599(96.46 \%)$, with $P$. falciparum - 21 (3.38\%), in 1 person was found two types of malarial parasites [2024].

In 1950, persons who have worked, in Stara Zagora region at malaria stations were: 4 doctors; 6 microscopists; 8 workersspecialists. Participating in the treatment and prevention of malaria (hininari): doctors - 7; paramedics - 4; midwives - 3; hygienist 2; administrators - 34. In 1950, in Stara Zagora region the lowest minimum temperature was recorded on January 12 $17.6^{\circ} \mathrm{C}$, and the highest maximum - August $19-36.5^{\circ} \mathrm{C}$. The average maximum temperature during the year was $16.2^{\circ} \mathrm{C}$ and the average minimum $-7.5^{\circ} \mathrm{C}$. The average maximum temperature during the malarial season was $23.8^{\circ} \mathrm{C}$ and average minimum $13.6^{\circ} \mathrm{C}$. The average temperature during the malarial season was $18.7^{\circ} \mathrm{C}$. In the malarial area of the county four types of malarial mosquitoes meet: A. maculipennis, A. superpictus, A. pseudopictus, A.bifurcatus [24].

\section{CONCLUSION}

Malaria is a disease that accompanies humanity, but in our days it continues to be severe and deadly socially important diseases. The European Centre for Disease 
Prevention and Control, the European Commission, the European Mosquito Control Association and WHO are working together to raise awareness of the problem and provide advice to countries on surveillance and control activities. Stronger malaria surveillance systems are urgently needed to enable a timely and effective malaria response in endemic regions, prevent outbreaks and resurgences, track progress and hold governments and the global malaria community accountable.

\section{REFERENCES}

1. Walsch K.C., Understanding Malaria and Lyme Disease., USA, 2010, pp. 87

2. Cox F.E., History of the discovery of the malaria parasites and their vectors. Parasites \& Vectors, 3 (1): 5, 2010.

3. Petrov P., Malaria. MF, Sofia, Bulgaria, 1990, 125 pp. (in BG)

4. Carter R., Mendis K.N., Evolutionary and historical aspects of the burden of malaria. Clin Microbiol Rev, 15 (4):56494, 2002.

5. Trihleb V.I., Malaria. The modern view on the problem. The report of the Scientific-practical conference with international participation "Topical infections: clinical manifestations, diagnosis, treatment and prevention" (Kiev 24-25.11.2010)

6. http://www.medicalnewstoday.com/artic les/150670.php

7. Sabatinelli G., Joergensen P., Majori G., Imported malaria in the WHO European Region 1971 - 1997. Giornale italiano di Medicina tropicale, 4: 1-5, 1999.

8. Sabatinelli G., Determinants in malaria resurgence in the former USSR. Giornale italiano di Medicina tropicale 4: 53-62, 1999.

9. WHO, 2015. Towards a malaria-free European Region by the end of 2015

10. Sabatinelli G., Ejov M., Joergensen P., Malaria in the WHO European Region (1971-1999). Euro Surveill., 6(4), 61-5, 2001.

11. Albu C., Brusin S., Ciancio B., Marrama-Rakotoarivony L., Sudre B., Van Bortel W., Warns-Petit E., Zeller H., ECDC surveillance report. Annual epidemiological report. Emerging and vector-borne diseases, 6-10, 2014.
CHAKAROVA B., et al.

12. Santa-Olalla Peralta P., Vazquez-Torres M.C., Latorre-Fandos E., Mairal-Claver P., Cortina-Solano P., A. Puy-Azón, et al. First autochthonous malaria case due to Plasmodium vivax since eradication, Spain, Euro Surveill.,15, 41, 14, 1-3, 2010.

13. Danis K, Baka A., A. Lenglet, W. Van Bortel, I. Terzaki, M. Tseroni,et al. Autochthonous Plasmodium vivax malaria in Greece, 2011. Euro Surveill., 16 (42), 2011.

14. Odolini S., Gautret P., Parola P., Epidemiology of imported malaria in the Mediterranean region. Mediter $\mathrm{J}$ Hematol Inf Dis., 4, 2012.

15. Romi R., Boccolini D., Menegon M., Rezza G., Probable autochthonous introduced malaria cases in Italy in 2009-2011 and the risk of local vectorborne transmission. Euro Surveill. 17, 2012.

16. Bruce-Chwatt C.J., de Zulueta J., The rice and fall of Malaria in Europe, University Press, Oxford, 1980.

17. Boeva - Bangyozova, V. Does returned malaria in Bulgaria? Forum Medicus, 12, 2012 (in BG).

18. Vuchev D. Tertian Malaria Outbreak Three decades after its Eradication. J. Infect. Dis. 54,79-80, 2001.

19. http://www.euro.who.int/en/countries/bu lgaria/news2/news/2015/05/bulgaria-50years-malaria-free

20. State Archives - Stara Zagora, F.272, op.1, a.e. 51, sheet 13-15 (in BG)

21. State Archives - Stara Zagora, F.272, op.1, a.e. 51, sheet 46-47 (in BG)

22. State Archives - Stara Zagora, F.272, op.1, a.e. 51, sheet 50-51 (in BG)

23. State Archives - Stara Zagora, F.272, op.1, a.e. 51, sheet 115-118

24. State Archives - Stara Zagora, F.272, op.1, a.e. 51, sheet 408-413

25. Kurdova R., D. Vuchev, P. Petrov. Epidemiological problems of malaria in Bulgaria, 1991 - 2000. Proceesing of the 1-th Balkan Conference on Malaria and Mosquito Control, Lithotopos-Serres, Greece, 2001.

26. Stanchev P., T. Todorov. Malaria in Bulgaria. MF, Sofia, Bulgaria, 1967, 100 pp. (in BG) 